\title{
DESPATRIARCALIZANDO: \\ JULIETA PAREDES Y SU VINCULACIÓN CON EL DISCURSO POLITICO Y POÉTICO DE MUJERES MAPUCHE.
}

\section{Depatriarchalizing: Julieta Paredes and its relation to the political and poetic discourse of Mapuche women.}

\section{CLAUDIA ARELLANO HERMOSILLA*}

\section{Fecha de recepción: 3 de junio de 2015- Fecha de aprobación: 10 de agosto de 2015}

\section{Resumen}

El siguiente texto explora la vinculación entre el discurso de Julieta Paredes -feminista indígena aymara, y una de las fundadoras del Colectivo Mujeres Creando Comunidad en Bolivia-, con algunas de las poetas mapuche, quienes sitúan el 'cuerpo' como topos de resistencia activa a la normatividad social, proponiendo no solo rupturas al canon tradicional, sino también realizando una crítica a la cultura patriarcal indígena y un cuestionamiento activo al feminismo occidental. Estos discursos van tensionando esa suerte de 'deber ser' que se transfiere a las mujeres de estos pueblos al representar una cultura en estado prístino, como expresión de aquello que se denomina tradición y que ha sido mantenido por la "colonialidad".

Palabras clave: feminismo indígena, patriarcado, decolonización.

\section{Abstract}

The following article explores the link between Julieta Paredes's speech - a feminist aymara woman and one of the founders of the Women Creating Community Group in Bolivia- with some of the mapuche poetesses, who establish the 'body' as a place of active resistance to social norms, proposing not only ruptures in the traditional canon, but also making a critique of the patriarchal indigenous culture and an active questioning of the western feminism. These speeches strain that sort of 'must be' which is transferred to the women of these peoples: when representing a culture in pristine condition, as an expression of what is called tradition, and it has been maintained by the "coloniality".

Keywords: indigenous feminism, patriarchy, decolonization.

* Antropóloga. Doctora en Estudios de Género, Universidad Paris VIII Saint Denis, Francia. Académica, Escuela de Antropología, Facultad de Ciencias Sociales, Universidad Academia de Humanismo Cristiano, Chile.

Correo-e: arellanohermosilla@gmail.com 


\section{Presentación}

Como supuesto que orientará el presente artículo, esbozo que los discursos tanto de dirigentas como de poetas mapuche, está estrechamente relacionado con los planteamientos de los movimientos despatriarcales y decoloniales que se han venido desarrollando en la última década en América Latina, especialmente en Bolivia a partir del Colectivo Mujeres Creando Comunidad, el que ha ido situando una resistencia activa en el desmontaje ${ }^{1}$ de discursos y prácticas patriarcales. A esto se suma el aporte del feminismo decolonial2 ${ }^{2}$, liderado por mujeres indígenas intelectuales y líderes políticas, que levantan un discurso de reivindicación propio y una crítica no solamente al feminismo occidental blanco, sino también a la estructura de poder denominada "colonialidad" (Quijano, 1992; Mignolo, 2000), la cual generó una "matriz de dominación" (Hill Collins, 2012) y con ella un sistema jerárquico con múltiples niveles de opresión que integra elementos estructurales, disciplinarios, hegemónicos y aspectos interpersonales.

Las voces de mujeres mapuche y la "emergencia del feminismo mapuche", no han sido ajenas a estas reivindicaciones, constituyendo propuestas de representación simbólica, desmontando el imaginario hegemónico -instalado desde el aparataje de la colonia ${ }^{3}$ y retomado por los Estados nacionales-, tensionando esa suerte de deber $\operatorname{ser}^{4}$ que se le transfiere a la(s) mujer(es) indígena(s).

Uno de los puntos interesantes en la política-poética $^{5}$ de mujeres mapuche, consiste precisamente en un ejercicio de deconstrucción de mitos o desmitificación, desarrollando una nueva consciencia del propio Yo y del Nosotros. Esto ocurre en discursos literarios y políticos que buscan, encuentran y defienden su propio lugar no en la dependencia respecto a las tendencias centralistas, sino encontrando su propia voz, en un tiempo y una memoria que no aparece registrada en la construcción de la supuesta identidad nacional.

Estos discursos, en especial la poética de mujeres, han intentado establecer una intervención critica de la cultura, invocando la apertura de lo que se encontraba obturado por el canon literario paternalista y centralista, "reclamos emancipatorios" de las diferentes comunidades, como lo llama Judith Butler, en tanto los que significan un reclamo son los propios "contaminados" y su lucha solo "reiniciará la dialéctica que produce su escisión y condición espectral" (2000:48). Este reclamo por el reconocimiento de la diferencia, estaría condenado por las cuotas de representatividad ${ }^{6}$ que le acomoda a la homogeneidad cultural e identitaria de la nación chilena. Solo un desvío de estos acuerdos podría asegurar la posibilidad de rearticulación y significación de un verdadero reconocimiento de las "diferencias culturales" (Bhabha, 2007), y de esta manera poder forjar una nueva historiografía de la memoria no contada de las mujeres indígenas. Al respecto, Ana Millaleo señala:

\footnotetext{
“(...) esto devela la poca sinceridad de la historiografía occidental y sus errores en la interpretación con otras culturas (...) desde los españoles a la actualidad, no se ha encontrado en condiciones de dar cuenta de lo que sucede en este espacio, que es solo 'descrito someramente', siendo solo la "esfera de lo público" (...) de los héroes masculinos de ese pueblo" (2011).
}

Como sabemos, mediante su poder instituyente las élites impusieron junto con su hegemonía política y su proyecto cultural un constructo identitario único de la nación y lo nacional, marginando del sistema cultural los discursos-otros de huachos, de indios y mestizos como agentes sociales y culturales disonantes. 
Se explica así, el empeño por releer y re-escribir por parte de mujeres indígenas, los documentos fundacionales de la época colonial, de la historia moderna o la transfiguración heroica de deidades religiosas o personajes históricos obliterados por la "cultura nacional" (Bhabha, 2007: 76).

No cabe duda que las propuestas de las feministas indígenas, desde la perspectiva de estudios decoloniales, con sus diferencias y matices, han abierto la posibilidad de que voces silenciadas empiecen a convertirse en referentes y en propuestas de pensamiento, cuestionando la lógica racionalista y progresista del universalismo del concepto mujer, que esconde dentro de sí, discursos de mujeres insatisfechas, al ser inventadas y construidas desde lo blanco, no incorporando lo real e imaginario de la raza, la etnia, la clase. Breny Mendoza, en su tesis sobre la "colonialidad de la democracia", establece que la democracia liberal existente en Occidente fue posible solo por la fusión entre raza y género. Comprendiendo este proceso es que llegamos a ver la confluencia del sistema heterosexista, el sistema de género colonial moderno con el capitalismo y la democracia liberal, que terminó beneficiando a las mujeres blancas de la metrópolis, que poco a poco lograron su inclusión en el pacto social de los hombres blancos mediante el privilegio heterosexual que se da a través del matrimonio y los beneficios extraídos de la conquistas de los derechos civiles, los cuales han dependido de la sobreexplotación de las mujeres negras, latinas e indígenas dentro de sus países (Mendoza, 2006).

Si tomamos en cuenta el reemplazo de estas prácticas y de estos discursos, marcados por las relaciones de poder y que nos reenvían a nociones foucaultianas como los dispositivos y la subjetivación, evitamos caer en el facilismo de considerar la situación colonial y poscolonial solamente en términos coercitivos, en donde la cultura indígena queda subordinada a la cultura dominante. La capacidad que tienen los dominados de utilizar en su propio beneficio y de incorporar a través de la subjetivación las prácticas y discursos de resistencia, han demostrado en las últimas décadas la vitalidad, de la resurgencia, reinvención y etnicidad estratégica de ir "más allá" para transformar lo establecido (Bhabha, 2007:87). De esta manera, las estrategias complejas en las relaciones de subordinación y de resistencia de las mujeres mapuche, por ejemplo, con el Estado chileno, llevan a cambiar de posiciones y a renegociar sus identidades, es decir, la nueva "reconstrucción de la génesis", consiste en "decolonizar" y "deconstruir" las historias de los pueblos originarios, poniendo en cuestión los mitos fundadores de la nación chilena y reemplazándolos por los contra-mitos (Memmi, 1985).

\section{Feminismo(s) indígena(s)}

Julieta Paredes, mujer aymara, feminista comunitaria, lesbiana, y una de las creadoras del "Movimiento Mujeres Creando" en Bolivia, plantea un discurso que tiene por objetivo deconstruir desde el lenguaje y la práctica, no solo emplazando rupturas al canon tradicional sino también una crítica que apunta a la cultura lesbofóbica y patriarcal aymara (Falconí, 2011:315). En su cuestionamiento al feminismo occidental de la igualdad, ella enuncia "yo, la peor de todas" (Paredes, 1996), reclamando la propiedad sobre sus propias etiquetas y evitando clasificaciones externas impuestas. Esta deconstrucción del lenguaje que realiza Julieta Paredes, va de la mano con una defensa lingüística de nombrar desde su propio tiempo y 
espacio, queriendo traducir el cuerpo femenino aymara desde distintos lugares o topos, es decir, desde la heterogeneidad andina.

En la escritura y discurso de Julieta Paredes, se expresa el encuentro entre el feminismo activista comunitario no occidental y la búsqueda por un nuevo lenguaje que se plasma en las categorías de descolonización y despatriarcalización ${ }^{7}$. Estas teorizaciones han sido ampliamente difundidas por ella y el colectivo que lidera -Mujeres Creando Comunidad-, instalando una problemática compleja al interior de los pueblos indígenas latinoamericanos, que apunta precisamente a un cuestionamiento directo a la "tradición y cosmovisión" de estas culturas. Paredes menciona que la ideología política del "patriarcado" es un "cuestionamiento abierto y lúdico al indigenismo. Una suerte de nuevo izquierdismo masculinista que quiere convencernos de que el machismo lo trajeron los invasores y que los hermanos indígenas estarían puros de cualquier patriarcado" (Paredes, 2010:3). Al parecer la autora busca "reapropiarse del poder estatal que cuestiona el mito, e ingresar al Estado de Derecho Boliviano" (Falconí, 2011:316). Por su parte, Ana Ñanculeo, feminista mapuche de la comunidad Calbul Llanquihuen señala que:

“(...) ahora las mujeres estamos recuperando la historia nuestra y el lugar que les pertenece a nuestras hijas (...) por eso es que nos quedamos pensando con más fuerza en desarmar el patriarcado y construir comunidades, sin instituciones" (Ñanculeo, 2008).

En esta línea, Julieta Paredes propone el concepto de "entronque de patriarcados", para señalar la existencia de un patriarcado originario en los pueblos del Abya Yala ${ }^{9}$ antes de la llegada de los colonizadores, como ella misma apunta: "el patriarcado es la primera estructura de dominación y subordinación de la historia, que funda el sistema de todas las opresiones. Es el sistema más poderoso y duradero de desigualdad, jerarquías y privilegios" (Paredes, 2014: 67).

La autora plantea la recuperación de la idea de comunidad dentro de los pueblos indígenas, para contraponer el individualismo del proyecto moderno y de los movimientos reivindicativos de corte occidentalista (Paredes, 2014). Por su parte, Rita Segato formula el análisis del "patriarcado de baja intensidad", indagando en cómo las relaciones de género van siendo modificadas históricamente por el colonialismo, partiendo del supuesto que las relaciones de género son preexistentes a la conquista (Segato, 2011). En relación a este cuestionamiento, Isolde Reuque, dirigenta mapuche, ya en el año 2002 cuando publicara su autobiografía Una Flor que renace, afirmaba que: "El machismo europeo se conjugó con el machismo propio mapuche (...) este machismo se demuestra con el celo y el mandato, esa superioridad del macho frente a la mujer" (Reuque, 2002: 227).

María Galindo, quien también fuera parte del Colectivo Mujeres Creando en Bolivia, apunta que "No se puede descolonizar sin despatriarcalizar". Galindo reivindica su autoría sobre el concepto de despatriarcalización en el contexto boliviano y lo pone a dialogar con el de descolonización, extendiendo la tensión entre ambos al horizonte del feminismo internacional. Galindo al igual que Paredes reclama:

“¿El patriarcado llegó a América con los colonizadores blancos? (...) no es cierto que el universo indígena no fuera patriarcal, porque el colonialismo otorgó a los hombres indígenas ventajas sobre las mujeres indígenas, porque un proceso serio de descolonización ha de deshacer esos privilegios patriarcales como parte del colonialismo y no inventarse una descolonización a su medida que inserta el sometimiento de las mujeres dentro de los saberes culturales ancestrales que deben ser preservados" (Galindo, 2013). 
La autora plantea que es necesario una revisión compleja sobre este tema ya que "no es solo la discriminación de las mujeres, sino la construcción de las jerarquías sociales, superpuestas unas sobre otras y fundadas en privilegios masculinos sobre las formas de organización social" (Galindo citada en Moreno, 2014). De esta manera, siguiendo a la autora: "La despatriarcalización se convierte entonces en la osadía de concebir al patriarcado como una estructura susceptible de ser des-montada" (Galindo, 2013). Desde la mirada mapuche, Isolde Reuque plantea intensas críticas a lo que ella denomina "caciquismo", entendido como el derecho que reclaman algunos hombres mapuche a tener múltiples mujeres, como signo de su tradición de "masculinidad guerrera" (2002: 20).

Aura Cumes, feminista maya, revisa por su parte, el debate cruzado entre el feminismo y el multiculturalismo "hegemónico", planteando una crítica frontal a las ideas que "absolutizan" a las mujeres y preguntándose si "¿podemos llamar patriarcado a la existencia de relaciones de género y opresión dentro de las comunidades originarias?" (Cumes, 2012).

De igual modo, la boliviana Silvia Rivera Cusicanqui apela al derecho como un "instrumento de la modernidad ilustrada de corte patriarcal" y el carácter falogocéntrico del sujeto concebido por la modernidad europea e impuesto al resto del mundo. Según Rivera Cusicanqui, la occidentalización y patriarcalización son procesos paralelos implantados lentamente por el modelo colonizador hegemónico, que ha contribuido a instalar las instituciones estatales a través de diferentes momentos de "modernización", y este modelo ha ido reconvirtiendo a las indígenas según los paradigmas del ser mujer europeo: de pastoras o tejedoras pasaron a ser consideradas fundamentalmente como madres, esposas, dependientes del varón (Rivera Cusicanqui, 2014). Por otro lado, Llanca Marín señala que:

"La invisibilidad, negación y exclusión del Estado chileno hacia las mujeres mapuche, que no cuenta con programas que involucren la situación ni nuestro modo de vida, también se traslada a gran parte del mismo Movimiento Mapuche. Influenciado por la ideología patriarcal, occidental y cristiana, ahora vemos cómo organizaciones mapuche se estructuran jerárquicamente, reproduciendo pequeños estados patriarcales, con autoridades superiores y comandadas principalmente por hombres, haciéndole el juego al Estado Nacional" (Marín, 2005).

De esta forma, se van articulando voces desde distintos rincones de América Latina, aquellos discursos reivindicativos sobre la despatriarcalización, a las cuales se le van sumando diversos colectivos y agrupaciones, intensificando así la propuesta latinoamericana "despatriarcal y decolonial". Ejemplo de esto es la Agrupación feminista lésbico-disidente mapuche "Segundo Patio", que trabaja en la Araucanía, cuyo objetivo político es:

“(...) la transformación de las prácticas sociales patriarcales, heterosexuales normativas, capitalistas y neoliberales desde la propia cotidianeidad (...) Nos paramos desde un Feminismo de lo cotidiano, y proponemos formas de vida, de criar y amar que estén fuera de la Heteronorma y del Patriarcado" (Segundo Patio, 2014).

\section{Des-haciendo el tiempo y ritmo lineal}

El mito entendido como símbolo fundacional, permite develar, en parte, cómo la ideología moderna liberal y racionalista logró instalar ideales normativos para conformar un reordenamiento de los territorios, de los tiempos, de los 
cuerpos y de los saberes. En este proceso de dominación la escritura realizó una cartografía expansionista, instalándose como la herramienta básica para reproducir el mito a partir de textos escritos. Este orden gramatical ${ }^{10} \mathrm{y}$ del control de la lengua de los pueblos dominados, llevó al reordenamiento simbólico, apoyado por el carácter sincrónico de la noción de tiempo lineal de la historia, "reordenando el espacio- tiempo de los pueblos colonizados” (Quijano, 2000).

La relectura del pasado no es unilineal en los procesos socio-históricos. Ya las reflexiones de Homi Bhabha acerca del discurso colonial y el sujeto, muestran cuán importante es la reescritura de los textos fundacionales, como él mismo señala "...Estamos obligados a reclamar la historia, nuestra memoria-tiempo" (Bhabha, 2007).

Julieta Paredes aboga por la des-colonización y la des-patriarcalización, que se fundan en des-hacer el tiempo lineal, tiempo instalado con la dominación colonial, oprimiendo el tiempo circular de los pueblos originarios, "se debe recuperar la memoria descolonizándola, denunciando sus lecturas y clasificaciones arbitrarias (...) descolonizar la temporalidad" (Paredes \& Guzmán, 2014:26).

Los pueblos andinos como la mayoría de los pueblos originarios de América no contaban con una cronología absoluta, ni narraciones totalizantes. Los conquistadores instalan una cronología y una ordenación tiempo-espacio, que buscó fijar subjetividades y jerarquías de control. Por tanto, la temporalidad circular propia de estos pueblos, queda sometida a la metrización.

Esta metrización, surge con la necesidad de anotar el ritmo ${ }^{11}$, el que inicialmente en las culturas ágrafas se transmitía de forma oral para poder recordarlo y transmitirlo. De esta manera, la metrización derivó en lo que hoy conocemos como "compás", que es "la manera de dividir y subdividir el tiempo musical oponiendo un orden periódico a la libertad del ritmo" (De Candé, 2002:77). Es decir, cada ritmo es único y tiene una organización propia, mientras que las agrupaciones o compases, que se pueden hacer del mismo no son hechos físicos sino construcciones adulteradas" (Berry, 1987: 302-303).

Si relacionamos la lógica del tiempo lineal (occidental) con el concepto de compás, y asociamos el tiempo circular con la concepción de ritmo ${ }^{12}$-entendiendo que el ritmo es subjetivo y se basa en las relaciones de intensidad y de duración de los sonidos, mientras que la métrica y el compás constituyen una división artificial (Souriau, 1998: 957). Por tanto, el gran logro de la dominación colonial fue precisamente que los pueblos colonizados entraran en la lógica del tiempo-compás, es decir, encasillaron al ritmo dentro de la métrica. Lo más seguro es que Franco de Colonia, no hubiese imaginado que esta métrica se extendería a una dominación cultural hegemónica de los posteriores pueblos colonizados. Octavio Paz, por su parte señala:

\footnotetext{
"La constante presencia de formas rítmicas en todas las expresiones humanas no podía menos de provocar la tentación de edificar una filosofía fundada en el ritmo. Pero cada sociedad posee un ritmo propio. O más exactamente: cada ritmo es una actitud, un sentido y una imagen del mundo, distinta y particular. Del mismo modo que es imposible reducir los ritmos a pura medida, dividida en espacios homogéneos, tampoco es posible abstraerlos y convertirlos en esquemas racionales. Cada ritmo implica una visión concreta del mundo (...) El ritmo, que es imagen y sentido, actitud espontánea del hombre ante la vida, no está fuera de nosotros: es nosotros mismos, expresándonos. Es temporalidad concreta, vida humana irrepetible" (Paz, 1972: 22).
} 
Entonces, una de las propuestas que instala Julieta Paredes con la idea de "descolonizar la temporalidad", es precisamente desmontar ese tiempo lineal -compás- instalado con la colonialidad. Se trata entonces de encontrar ese ritmo-tiempo, en donde cada quien o cada pueblo o cultura se despliegue a su propio ritmo o movimiento, a ese fluir que fue negado $y$ en el cual nuestros pueblos originarios quedaron supeditados.

\section{La performance del cuerpo y el activismo político}

Una de las estrategias que se plasma en algunos de los discursos del feminismo indígena, y el de algunas poetas y escritoras mapuche, ha sido posicionar el cuerpo como acto político de rebeldía, ubicando justamente la producción teórica sobre el cuerpo abyecto para articular una reflexión pendiente, que fueron los cuerpos expropiados de las mujeres dentro de la historia de colonización geopolítica y discursiva del continente (Espinosa, 2010). Esto lo podemos apreciar en la construcción discursiva de Julieta Paredes y su texto "Mirando al signo", donde ella escribe: "Nuestras sociedades son cuerpos como mi cuerpo, en los cuales hay heridas, cicatrices y zonas erógenas" (Paredes citada en Ayllón, 1999:160). Paredes, invoca la marca visual o cicatriz producto de la herida colonial, señalada por Mignolo. Pero luego visualiza la carne, el deseo a través de estas "zonas erógenas", visualizando el goce. De esta manera el cuerpo, se vuelve un lugar privilegiado en el cual se plasma el discurso escritural y la acción política, como en la escritura poética de Roxana Miranda Rupailaf, quien con el objetivo de poder transgredir el orden establecido, va trazando las fronteras internas de su Yo, fragmentos de su identidad híbrida, mestiza, huilliche, conectándolas y reescribiéndolas con el relato hegemónico del mito judeo-cristiano y de la colonialidad. Lo que hace Roxana es "no olvidar", no olvida el pasado colonizador. Por eso la poeta realiza un "montaje" de la historia: corta, pega, vuelve a montar, remonta al origen, al paraíso, develando la "descolonización de la intimidad":

\section{Serpientes de Sal}

Come la manzana mi querida.

Suelta baba -rojo en las dos llamas.

Muérdete a la carne y haz el jugo espeso mezclándolo con sal. Devórate los frutos en fuego ${ }^{13}$ y muéstrale el deseo a los que duermen.

(Del libro La seducción de los venenos, 2008)

Miranda Rupailaf suscita el deseo, ese "doloroso deseo" que las mujeres llevamos encarnado. Es interesante señalar como se funda en el mito el movimiento del mal en el deseo, y de cómo este afectará al objeto, a la mujer, al que la sexualidad le asignara un propósito que no será temporal, puesto que el deseo la perseguirá por siempre. Este "deseo" es el mal que está en ella, estableciendo la sospecha o el miedo que la mujer suscita (Rousseau-Dujardin, 2006:18). La variante que desarrolla Roxana Miranda, el "peligroso deseo", deja de estar apegado al dolor de la mujer, como lo dicen los proverbios. Aquí la imagen es la subversión del placer, es decir, aquí el "deseo" transgrede los mitos fundadores. 
En este sentido ese poner el cuerpo en su discurso -como transgresión-, es el compromiso ético y político, teniendo como objetivo desordenar el orden establecido desde la centralidad que insiste en la carne, cuerpo controlado y abyecto, pero que puede ser resignificado. El estudio realizado por Michael Horswell en el área andina, ha revelado, por ejemplo, el uso del cuerpo como un archivo censurado por los vestigios de la religiosidad cristiana (Horswell, 2005: 34). El cuerpo antes y después de la llegada de los españoles fue un poderoso archivo de la memoria, como lo ha sido la literatura oral como medio de preservación de las tradiciones.

Estas ideas demuestran la importancia de la existencia de un cuerpo situado en el espacio, rechazando, de esta forma, la idea universal del discurso feminista blanco. Lo "universal" sería una especie de absolutismo, de un todo, que refleja también el triunfo de la razón sobre la naturaleza: "Lo universal se propone como una estrategia de dominación y colonización de los cuerpos y las mentes (...) lo universal entonces es uno, y no es neutro, es una estrategia de dominación" (Paredes \& Guzmán, 2014:20). Como vemos, estos discursos tratan precisamente de abandonar algo, de desprenderse de esas categorías impuestas dentro de ese ordenamiento colonial (blanco/indio) donde además, "lo mestizo" queda relegado, omitido.

María Galindo del colectivo Mujeres Creando, se pregunta:

\footnotetext{
“¿Será imperdonable hablar del cuerpo y del placer en un país de hambre, autoritario y violento como es hoy el rostro de Bolivia? ¿Será imperdonable que una india hable de recuperar su cuerpo con la misma pasión que habla de recuperar su tierra?" (Galindo, 2006:27).
}

Este poner el cuerpo, como lo señalan Paredes, Miranda Rupailaf y Galindo, también está estrechamente vinculado al linaje femenino. Esta memoria genealógica según Paredes se presenta desde el ombligo: "el ombligo nos conecta siempre con la otra, que es la madre, y desde esa relación inicial siempre estamos construyendo y creando el diálogo en comunidad" (Paredes, 2010: 7). Julieta Paredes recuerda que su abuela le enseñó a apreciar su propia diferencia corporal como un sitio de orgullo: "Aprendí de mi abuela que la sociedad eran cuerpos donde el cuerpo de ella y mi cuerpo eran otros cuerpos" (Paredes, citada en Ayllón, 1999: 156). La idea de Paredes es recuperar la lucha de nuestras 'ancestras': "Manuela Condori, Isabel Wallpa, Tomasina Silvestre, Isidora Katari, Bartolina Sisa, Gregoria Apaza y muchas otras que encabezaron los levantamientos indígenas anticoloniales en 1781" (Paredes \& Guzmán, 2014: 28).

Este discurso interpela - al igual que en la poética de mujeres mapuche- la memoria genealógica del linaje femenino, como lo podemos apreciar en el poema "Walinto", de Graciela Huinao (2008):

\section{Mi abuela}

Almerinda Loi Katrilef se levanta de esa tierra a esculpir golpe a golpe una macabra escultura:

Tres chilenos

embistiendo a caballo

a una mujer williche en su octavo mes de embarazo.

Hacha en mano abuela defendiste tu tierra.

Cerraron tus cicatrices y yo abro este poema..." 
La retórica de Huinao, apela al "nosotras" desde la línea matrilineal. Son las abuelas que hablan a través de ellas y de otras mujeres que fueron antes que ellas.

\section{El arte de Hilar y el guiño escritural}

En el libro Hilando Fino. Desde el feminismo comunitario (2010), podemos ver el guiño escritural con el trabajo que hicieran las poetas mapuche el año 2006 en Chile, en el primer "Encuentro de poetas mujeres mapuche" que lanzó la publicación de la primera antología de poetas mujeres mapuche, Hilando en la memoria $(2006)^{14}$, seguido por Hilando en la memoria, Epu rupa (2009). El proyecto tuvo por objetivo generar un espacio entre poetas que fuera colectivo, multi-medial, transgeneracional y social, integrando la dimensión política de la poesía mapuche escrita por mujeres, para dialogar en torno a la memoria, la traducción, el cuerpo y la poesía como escritura de resistencia. Las dos antologías publicadas logran producir un nuevo imaginario que eclipsa con el imaginario chileno marcado por la invisibilidad y la discriminación de las voces mapuche (Goñi, 2014).

Ambos textos, Hilando Fino e Hilando la memoria, hacen referencia al arte de hilar, que es el traspaso del conocimiento del telar, propio de las relaciones entre mujeres, y que el ejercicio de este conocimiento se mantiene y perpetúa por medio de un pacto sagrado entre las mismas, produciendo reconocimiento de solidaridad y de seguridad, creando de esta forma sus propias representaciones de la realidad, y estableciendo lo que el feminismo de la diferencia llamó affidamento ${ }^{15}$.
Ana Millaleo, socióloga mapuche en su texto "El Witral, la Escritura Ancestral de las Mujeres Mapuche", señala que el pueblo Mapuche no conocía la escritura ni la lectura, que la transmisión cultural mapuche se hace por medio de la oralidad, lo cual significa que la historiografía ha negado la existencia de otro tipo de escritura:

\footnotetext{
"Uno de los temas que han sido poco investigados es lo de la escritura ideográfica por medio del telar, escritura (dato no menor) que era manejada principalmente por las mujeres mapuche. La invisibilización de estas temáticas se inscriben principalmente en la centralidad que se le da en la historiografía occidental a los hombres mapuche, principalmente a la utilización de la figura del héroe para la construcción de los estados nacionales, a la asociación de lo indio a la barbarie y al reduccionismo del arte mapuche a la artesanía" (Millaleo, 2011).
}

Esta memoria femenina indígena e invisibilizada, de la que habla Millaleo, no solo se representa en el arte, sino también en el activismo histórico que han tenido las mujeres indígenas, y que ha sido sistemáticamente invisibilizada ${ }^{16}$.

Estos discursos del(los) feminismo(s) indígena(s) -con sus continuidades y discontinuidades-, han ido encontrando a nivel representacional "un lugar" que les posibilita hablar por sí mismas, pero con las dificultades de cargar con una lengua y una cultura que no se asumen como propias. Entonces la pregunta es: ¿Cómo escribir de nuevo, si lo que ha sido escrito correctamente y aprendido fue de manera incorrecta mil y una veces a través de la historia? Esa es la difícil labor de la escritura: dar en el lenguaje del mundo los mensajes que vienen de las profundidades, y que desafían la palabra.

En este sentido, Silvia Rivera Cusicanqui habla de una "artesanía teórica", la cual hace referencia, desde la sociología de la imagen, 
a una forma de descolonizar la mirada, lo que implica entrar a observar desde la propia biografía, sin el método rígido y paralizante de la observación científica occidental. Esta "artesanía teórica" pretende recuperar la confianza en la propia mirada desde lo aymara:

\footnotetext{
“(...) quienes viven en una comunidad, pueden hacer una investigación magnífica que ningún antropólogo podría, pero lo coartan porque debe leer a autores [europeos] que no comprende (...) revertir la relación de autoridad pedagógica para convertir al productor de conocimiento en un sujeto de pleno derecho, con confianza en sus propias ideas, en su propio lenguaje y hasta en sus propios silencios" (Rivera Cusicanqui, 2014).
}

La idea de la autora consiste en confiar en la propia mirada y recuperarla, desarrollando de esta manera la descolonización del lenguaje y así poder describir y escribir desde la propia cosmovisión.

En este sentido Adrienne Rich (1991), explora el poder que el lenguaje poético tiene dentro de la política y cómo este poder de lenguaje tiene repercusiones dentro del discurso feminista y que se extiende fuera de ello para tener repercusiones dentro de la sociedad. Al igual que la política, la poesía es siempre subjetiva y conlleva diferentes interpretaciones; en este sentido, el lenguaje político está ligado íntimamente con la política cultural. Este paralelismo entre la subjetividad de la poesía y la subjetividad de la política puede ser una razón por la cual ciertas escritoras optan por la poesía para proyectar sus mensajes feministas. Rich, señala que la poesía tiene un poder político que puede efectuar cambios dentro de la sociedad. Al examinar las razones porque muchas escritoras indígenas feministas optan por la poesía, sugiere que estas escritoras no emplean la poesía simplemente por escapar del discurso dominante, sino ellas intentan llevar algo nuevo a este género: es una manera de cambiar el orden establecido.

Poco a poco, desde distintas esquinas, se comienzan a juntar las voces de mujeres indígenas, haciendo alusión al "recuperemos el nosotras", recuperar esos cuerpos de manera colectiva, comunitaria y territorialmente (Mujeres Creando, 2005). Como vemos, estos discursos "critican las escrituras que colonizan discursivamente las heterogeneidades materiales e históricas de las vidas de las mujeres del tercer mundo produciendo/representando así una mujer del tercer mundo" (Mohanty, 2008: 33). Dando paso de esta forma, a lo que denomino como "poéticas del abandono", basada en una expresión enunciativa del cuerpo-mujer que desmonta estructuras de pensamiento, de creencias o de sentimientos fabricados por el discurso falogocéntrico, generando la deconstrucción y posterior resignificación de los mitos fundadores, como pérdidas voluntarias para abrir espacios obliterados de la historia.

Si instalamos el conocimiento de las mujeres indígenas de las diferentes culturas y pueblos de nuestro continente, constituyéndose en fuentes de saber nombrado, situado, válido y con autoría propia, podremos conectar el deseo del cuerpo y el cuerpo social, entretejiendo espacios legítimos de reivindicación, donde el discurso se ve a sí mismo socialmente conectado a un colectivo más grande, percibiendo la intencionalidad de crear un movimiento indigenista feminista Sur-Sur. Si nos atrevemos, podemos abrir otros puentes y mover otros límites.- 
Notas

${ }^{1}$ Si Walter Benjamin desplegó las estrategias del "montaje" para pensar tiempos de guerra, crisis y quiebre de sentidos, parece adecuado plantear la pregunta por la pertinencia de este concepto para pensar la imagen fabricada de las mujeres indígenas, en tanto imagen arrancada del pasado y que remite a antiguos símbolos y totalidades ahora resquebrajadas. Para que esta tarea de representación no aparezca naturalizada es que exhibo su carácter de artificio y su dimensión política. Esto supone explorar y sumergirse en las fronteras de lo imaginario entre cuerpo y territorio, para que podamos encontrar esos nuevos sentidos de ciudadanía simbólica y práctica de nuevas identidades.

2 Entre las feministas de esta línea, podemos mencionar a Silvia Rivera Cusicanqui, Aura Cumes, Leyla Gonzáles, Marisol de la Cadena, Julieta Paredes, María Lugones, Ochy Curiel, Breny Mendoza, Rita Segato, Sueli Carneiro y Chuy Tinoch, entre otras.

${ }^{3}$ Se considera como manifestación de lo poscolonial no solamente lo escrito después de la colonia, sino un corpus mucho mayor que se extiende desde el momento de la colonización hasta el presente. De acuerdo a los críticos Coronil, Quijano y Mignolo, la producción de textos poscoloniales comienza en el momento de la colonización y todos los textos que resisten la empresa colonizadora se incluyen en este corpus. Es decir, las fronteras de lo que se considera poscolonial se ven expandidas para incluir los debates sobre la alteridad y sus representaciones.

${ }^{4}$ Las innumerables investigaciones centradas en el rol que han cumplido las mujeres indígenas, se han enfocado en su mayoría, en la búsqueda de un imaginario "esencialista del ser mujer", basándose en el rol de la reproducción de la cultura, o bien en las articulaciones entre discursos de resistencia como forma de diferenciación de la cultura nacional.

${ }^{5}$ Entendiendo esta política-poética, como "el poder que el lenguaje poético tiene dentro de la política y cómo este poder de lenguaje tiene a la vez, repercusiones dentro del discurso feminista, y que se extiende fuera de ello para tener repercusiones dentro de la sociedad" (Rich, 1991).

${ }^{6}$ Estas cuotas de representatividad, se vieron claramente reflejadas en Chile a la vuelta de la democracia en la década de 1990, con la difusión y publicación de textos de autores mapuche, anunciando y celebrando la consigna de "diversidad nacional". Y, sin ir más lejos, con el Premio Nacional de Literatura otorgado el presente año a Isabel Allende, ratifican estas cuotas. Este premio que nace el año 1942, lo han recibido solo 4 mujeres hasta la fecha: Gabriela Mistral (1951), Marta Brunet (1961), Marcela Paz (1982) e Isabel Allende (2010). Y para señalar que esto no solo ocurre en Chile, en Francia el Premio Literario Goncourt, que se inaugura en 1903 , solo lo han recibido 7 mujeres en ¡108 años!

${ }^{7}$ Desde año 2009, Bolivia se transforma en un Estado Plurinacional. En esta nueva Constitución, se instala el Ministerio de Cultura y Turismo, que cobija al Viceministerio de Descolonización y Despatriarcalización.

${ }^{8}$ Paredes señala que las mujeres indígenas han ido de mejor en peor en sus derechos, a diferencia de las mujeres occidentales que han ido de peor a mejor. Como ejemplo de ello, la autora plantea que las mujeres aymara antes de la conquista tenían derecho a la tierra: Los hombres heredaban un tocapu y las mujeres medio tocapu, pero no heredaban igual cantidad.

${ }^{9}$ Abya Yala es el nombre dado al continente americano por el pueblo Kuna de Panamá y Colombia antes de la llegada de los europeos, y significaría "tierra en plena madurez o tierra de sangre vital". En López Hernández, M. Á. (2004). Encuentros en los senderos de Abya Yala (1 ${ }^{\underline{a}}$ ed.), p. 4.

${ }^{10}$ La Gramática de la Lengua Castellana de 1492 de Elio Antonio de Nebrija, es una obra fundamental en la historia del pensamiento lingüístico hispánico, ya que es la primera gramática de la lengua cuyo propósito, además de fijar la lengua castellana y su pervivencia, era colonizar y dominar a los nuevos territorios conquistados.

${ }^{11}$ Los primeros sistemas de notación empleados para plasmar el canto gregoriano eran bastante primitivos y solo indicaban la dirección ascendente o descendente de la voz. Esto también se debía a que aquella música se caracterizaba por su ritmo libre determinado por la palabra. Con el tiempo, surgió una notación basada en modos rítmicos que fue desarrollada por Franco de Colonia, compositor de la Escuela de Notre Dame, entre 1215 y 1270, quien sustituyó al ritmo aún no medido, impulsando el concepto de métrica o medida de la música, paso previo elemental para poder constituir una agrupación de tiempo mayor, es decir, el compás. De esta forma, el tempo, hace referencia a la velocidad con que debe ejecutarse el ritmo. En Abromont, C. (2005). Teoría de la música. p. 148-153

${ }^{12}$ La naturaleza del ritmo es primordialmente subjetiva. La idea de regularidad define el ritmo, pero no es la única ya que una de las primeras definiciones de ritmo en la historia de la música está relacionada con su raíz griega (rheos, fluir), marcando así una relación directa con el movimiento. El ritmo también es la cronología de los acontecimientos a escala humana, de los sonidos musicales y los silencios, de los pasos de una danza o el tiempo del lenguaje hablado y la poesía. En Snyder, B. (2000). Music and memory, p. 161

${ }^{13}$ El simbolismo del fuego, aquí es interpretado como transformación, asociación a la líbido y a la fecundidad, en tanto purificación y a la vez erotismo.

${ }^{14}$ En la primera antología Hilando la memoria (2006), participaron siete poetas mapuche: Graciela Huinao; Adriana Paredes Pinda; Roxana Miranda Rupailaf; María Isabel Lara Millapán; Faumelisa Manquepillán; Maribel Mora Curriao y María Teresa Panchillo. En la segunda edición del libro Hilando en la Memoria, Epu rupa (2009) participaron catorce poetas mapuche: María Isabel Lara Millapán; Graciela Huinao; Jacqueline Caniguan ; Faumelisa Manquepillán; Roxana Miranda Rupailaf; María Huenuñir; Adriana Paredes Pinda; Karla Guaquin; Alejandra Llanquipichun; Eliana Pulquillanca; Jeanette del Carmen Huequemán; María Elisa Huinao; Juana Miriam Lancapichun y María Teresa Panchillo. 
${ }^{15}$ El concepto es forjado por el Colectivo de la Librería de Mujeres de Milán y se refiere al reconocimiento mutuo de la autoridad de las mujeres, para sostenerse entre ellas y para hacer causa común frente al poder patriarcal.

${ }^{16}$ Francisca Quilaiqueo señala: "La mujer mapuche ha peleado desde siempre por sus derechos y dignidad". Podemos nombrar algunos casos emblemáticos del activismo mapuche actual: Patricia
Troncoso, prisionera política mapuche quien estuvo 55 días en huelga de hambre al interior de la cárcel a fines del 2003, como también las ñañas Quintreman que en territorio Pehuenche dieron muestra de firmeza y dignidad en contra del megaproyecto de la represa Ralco, o María Huenchún de la comunidad de Boyeco que el año 2002 luchó para erradicar un basural en su territorio.

\section{Referencias bibliográficas}

Abromont, C. (2005). Teoría de la música. México: Fondo de Cultura Económica.

Ayllón, V., Prada, A., \& Contreras, P. (comps.). (1999). Diálogos sobre escritura y mujeres. Memoria. La Paz: Sierpe.

Benjamin, W. (2005). Libro de los pasajes, Madrid: Akal (1973). Poesía y capitalismo. lluminaciones II. Madrid: Taurus.

Berry, W. (1987). Structural Functions in Music. Courier Dover

Bhabha, H. (2007). Les Lieux de la Culture. Une Théorie Postcoloniale. Paris: Payot.

Butler, J., Laclau, E., \& Žižek, S. (2000). Contingencia, hegemonía y universalidad. Diálogos Contemporáneos en la Izquierda. Buenos Aires: Fondo de Cultura Económica.

De Candé, R. (2002). Nuevo diccionario de la música, Vol. 1. Grasindo.

Derrida, J. (2006). "La violencia de la letra: de Lévi-Strauss a Rousseau". Revista Observaciones Filosóficas. Libros y recensiones.

Falabella, S., Ramay, A., \& Huinao, G. (eds.). (2006). Hilando en la memoria. 7 mujeres mapuche. Santiago: Cuarto Propio.

Falconí, D. (2011). De las cenizas al texto: Transgresiones identitarias gays, lesbianas y queer en el ordenamiento literario andino contemporáneo. (Tesis doctoral para obtener el grado académico de doctor en literatura comparada). Universidad Autónoma de Barcelona, Barcelona.

Hill Collins, P. (2012). "Rasgos distintivos del pensamiento feminista negro". En Jabardo, M. (ed.). Feminismos negros. Una antología. Madrid: Traficantes de Sueños.

Huinao, G. \& Miranda Rupailaf, R. (eds.). (2009). Hilando en la memoria. Epa rupa. 14 mujeres mapuche. Santiago: Cuarto Propio.

Galindo, M. (2006). "Indias, putas y lesbianas juntas, revueltas y hermanadas ¡Un libro sobre Mujeres Creando!”. En Monasterio, E. (ed.). No pudieron con nosotras. El desafío del feminismo autónomo de Mujeres Creando. La Paz: Plural.

Goñi, A. (2014). "Voces de Mujeres mapuche. Primera antología de poetas mujeres mapuche: Hilando en la memoria". Disponible en https://comunicacionesantropologia.wordpress.com/2014/07/28/ voces-de-mujeres-mapuche-primera-antologia-de-poetas-mujeresmapuche-hilando-en-la-memoria/ Fecha de consulta: 16 de marzo de 2015.

Horswell, M. (2005). Decolonising the Sodomite. Queer Tropes on Sexuality in Colonial Andean Culture. Austin: University of Texas Press.

Huinao, G. (2008). Walinto. Santiago: Cuarto Propio.

López Hernández, M. (2004). Encuentros en los senderos de

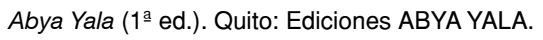

Mignolo, W. (2000). "La colonialidad a lo largo y a lo ancho: el hemisferio occidental en el horizonte colonial de la modernidad". En Lander, E. (comp.). La colonialidad del saber: eurocentrismo y ciencias sociales. Perspectivas Latinoamericanas. Buenos Aires: CLACSO.

Millaleo, A. (2011). "El witral, la escritura ancestral de las mujeres mapuche". Mapuexpress, 15 de marzo. Disponible en: http://servindi. org/actualidad/41425 Fecha de consulta: 28 de marzo de 2015.

Miranda Rupailaf, R. (2008). La seducción de los venenos. Santiago: LOM.

Mohanty, Ch. (2008). "Bajo los ojos de occidente". En Estudios postcoloniales. Ensayos fundamentales. Madrid: Traficante de Sueños

Mujeres Creando. (2005). La virgen de los deseos. Buenos Aires: Tinta Limón. (2002). Porque la memoria no es puro cuento. La Paz: Mujeres Creando. (1999). Grafiteadas. La Paz: Mujeres Creando.

Marín, LI. (2005). "La matria mapuche". En Azkintuwe Noticias, 8 de marzo. Disponible en: http://www.mujeresenred.net/spip. php?article1359 Fecha de consulta: 8 de noviembre de 2014.

Paredes, J. (2010). Hilando Fino. Desde el feminismo comunitario. La Paz: Mujeres Creando Comunidad.

(1996). Del mismo barro. La Paz: Mujeres Creando.

Paredes, J. y Guzmán, A. (2014). El tejido de la rebeldía. ¿Qué es el feminismo comunitario? Bases para la despatriarcalización. La Paz: Mujeres Creando Comunidad.

Paz, O. (1972). "El ritmo". En El arco y la lira. México: Fondo de Cultura Económica.

Quijano, A. (2000). "Colonialidad del poder, eurocentrismo y América Latina". En Lander, E. (comp.). La colonialidad del saber: eurocentrismo y ciencias sociales. Perspectivas Latinoamericanas. Buenos Aires: CLACSO. 
Quilaiqueo, F. (2012). "Mujer, pueblo y cultura mapuche". Ponencia presentada en el Seminario "Academia Mapuche", Colonia (Köln), Alemania, octubre. Disponible en: http://www.mapuche.nl/doc/ francisca_quilaqueo121012.pdf Fecha de consulta: 12 de marzo de 2015.

Reuque, I. (2002). Una Flor que renace. Autobiografía de una dirigenta mapuche. Santiago: DIBAM.

Rich, A. (1991). What Is Found There: Notebooks on Poetry and Politics. Nueva York: Columbia UP.

Rivera Cusicanqui, S. (2014). "Si hay un territorio colonizado es el lenguaje”. Revista Diálogos con las Ciencias Sociales, № 1. FACSO, Universidad de Chile.

Rousseau-Dujardin, J. (2006). Orror di Femmina. La peur qu'inspirent les femmes. Intempestives, Saint-Denis, Paris : Presses Universitaires de Vincennes.
Segundo Patio. Colectivo Feminista de la AraucaníaTemuco. Disponible en: http://segundopatiolabanda.wix.com/ segundopatio\#!quienessomos/c161y Fecha de consulta: 20 de marzo de 2015.

Snyder, B. (2000). Music and Memory. Cambridge: MIT.

Souriau, É. (1998). Diccionario Akal de Estética. Madrid: Akal.

Spivak, G. (2009). Muerte de una disciplina. Palinodia: Santiago.

Vera, A. (2014). "Moral, representación y "feminismo mapuche": elementos para formular una pregunta". Polis, Vol. 13, № 38 . Santiago. Disponible en: http://www.scielo.cl/scielo.php?script=sci_ arttext\&pid=S0718-65682014000200014\&lng=es\&nrm=iso\&tlng=es Fecha de consulta: 20 de marzo de 2015. 